\title{
TEMPOROMANDIBULAR JOINT CAPSULAR AND PERICAPSULAR INJECTION OF SCLEROSING AGENT AND ITS EFFECT ON THE MOUTH OPENING (AN EXPERIMENTAL STUDY)
}

\author{
Linda A. ElRahmany ${ }^{1} B D S$, Sherief H. Elghamrawy ${ }^{2} P h D$, Nevein S. Mohamed ${ }^{2} P h D$, Hanaa S. \\ $\operatorname{Raslan}^{3} P h D$.
}

ABSTRACT

INTRODUCTION: Recurrent Temporomandibular Joint (TMJ) dislocation is a relatively common problem encountered in the oral and maxillofacial clinics. Multiple interventions and injections had been described in the literature for TMJ dislocation treatment. Ethanolamine Oleate is a sclerosing agent that can be used in the treatment of TMJ dislocation by creation of inflammatory reaction which induces fibrosis, formation of adhesion and scar formation in the surrounding soft tissues.

OBJECTIVES: The aim of this study was to evaluate Ethanolamine Oleate injection in TMJ capsular and pericapsular tissue and its effect on the mouth opening in rabbits.

MATERIALS AND METHODS: This experimental study was conducted on eight New Zealand rabbits (approximately 6 months old and weight 2-3 Kg). They were divided into two groups: Group One (Study Group): Eight of these rabbits were injected by Ethanolamine Oleate into their Temporomandibular Joints capsule and pericapsular tissues in the right side. (Group two): left side was acting as a control group. Each two rabbits were sacrificed at intervals of two, four, six and eight weeks. TMJ capsule and pericapsular tissues were dissected and prepared to evaluate the histopathologic changes.

RESULTS: The pericapsular tissue area surrounding the Temporomandibular Joint area showed similar histopathologic aspects in all samples. In fact, the pericapsular area showed angiogenesis with presence of fiber, new formed bone and cartilaginous hyaline tissue confirming the diagnosis of fibroblastic activity followed by zone of endochondral ossification and trabecular bone mixed with bone structures.

CONCLUSIONS: Ethanolamine Oleate injection is a safe, simple, cost effective treatment of TMJ dislocation with minimal post-operative complication and foreign body reaction.

KEYWORDS: TMJ, Dislocation, Sclerosing.

1 -MSc Oral and Maxillofacial Surgery, Faculty of Dentistry, Alexandria University, Egypt.

2- Professor of Oral and Maxillofacial Surgery, Faculty of Dentistry, Alexandria University, Egypt.

3- Professor of Oral Pathology, Faculty of Dentistry, Alexandria University, Egypt.

\section{INTRODUCTION}

Temporomandibular disorders (TMDs) are that group of pathologies that affect TMJ, muscles of mastication and related structures $(1,2)$.

They are classified into non-articular and articular disorders that may range from a little temporomandibular dysfunction to complete TMJ dislocation (3).

TMJ dislocation is considered one of the most serious TMJ disorders when the condyle travels down and anterior to the articular eminence; it may be unilateral or bilateral, non-reducible (acute form) or reducible (chronic or habitual form (4).

Acute temporomandibular joint dislocation is usually managed by manually pressing the mandible downwards and then pushing it back upwards in an attempt to try relocating the condyle in the glenoid fossa (5). If the condyle continues to dislocate several times, it is described as chronic recurrent TMJ dislocation. Chronic recurrent TMJ dislocation may occur as a result of everyday activities such as yawning and laughing. Also, it may occur after excessive mouth opening during dental treatment and general anesthesia procedures $(6,7)$.
The pathogenesis of chronic recurrent TMJ dislocation is attributed to a combination of factors including laxity of the TMJ ligaments, weakness of the TMJ capsule, an unusual eminence size or projection. Muscle hyperactivity or spasms, trauma, and abnormal chewing movements that do not allow condyle to translate back (8).

Different surgical and nonsurgical methods have been used for the treatment of TMJ hypermobility. The conservative methods include physiotherapy, occlusal splints, and avoiding activities that cause largemouth opening $(5,9,10)$.

Minimally invasive methods include injections of sclerosing agents, intra-articularly and extra-capsularly or injection of botulinum toxins in the surrounding muscles ( 6 , $11,12)$.

Autologous blood injection in the TMJ for the treatment of hypermobility is a simple technique, but for unclear reasons, it was not popular and only recently was reintroduced by Hasson and Nahlieli $(4,5,7)$.

The sclerosing agents most widely used are $5 \%$ sodium Morruhate and 5\% Ethanolamine Oleate (EO) (13).

Ethanolamine Oleate is a sclerosing agent which has been approved by FDA for the treatment of varicose veins 
and esophageal varices. It is a minimally invasive, simple, effective technique. It is less likely to cause allergic reactions than sodium morrhuate or sodium tetradecyle sulphate (14).

The aim of this study was to inject a sclerosing agent into the TMJ capsular and pericapsular tissues to observe the effect of this drug into the TMJ capsular and pericapsular tissues with the hypothesis that the $\mathrm{EO}$ is an effective agent in TMJ recurrent dislocation treatment.

\section{MATERIALS AND METHODS}

Ethical clearance was obtained from the university ethical committee.

\section{Experimental animals}

This study was conducted on eight New Zealand rabbits; about six months old, with average weight 2 to 3 kilograms. The animals were kept under the same nutritional and environmental conditions in the animal house, the Physiology Department, Faculty of Medicine, Alexandria University.

Rabbits were divided into two groups:

Group I (Study Group): include eight rabbits which were injected by Ethanolamine Oleate sclerosing agent in TMJ capsular and pericapsular tissue in the right side.

Group II (Control Group): include eight rabbits (left side of the same rabbits) were acting as a control group. (Control group only taking as a reference of normal histological tissues).

\section{Materials}

Ethanolamine Oleate (Ethanolamine Oleate 5\%)

Ethanolamine Oleate (Ethamolin) (Ethanolamine Oleate EIPICO, Cairo, Egypt) is a sclerosing agent. It is composed of ethanolamine and oleic acid. It was supplied as a sterile clear, straw to pale yellow colored solution in $5 \mathrm{ml}$ glass ampoules.

\section{Methods}

\section{I- Preoperative care}

All rabbits were healthy as documented by a veterinarian report. The rabbits were kept under the same nutritional and environmental conditions and were kept on the same balanced diet throughout the whole period of the study.

\section{II- Surgical procedures}

\section{1- Anesthesia}

All operating procedures were performed under general anesthesia and sterile conditions in an animal theatre. Each animal was generally anaesthetized via intravenous injection of Thiopentone Sodium (Intra-val, Egyption Int. Pharmaceutical Industries Co. (E.I.P.CO.)-Egypt), the dose of which was calculated on the basis of $30 \mathrm{mg} / \mathrm{kg}$ body weight.

\section{2- Surgical operation}

Manual jaw movements were done to palpate the condylar head in front of the ear and allocate the site of injection. The pre-auricular area was prepared by using povidone Iodine (Betadine: The Nile Co, for pharmaceuticals \& chemical industries (A.R.E) under license from Mundipharma-AGBasal-Switzerland) solution for sterilization and shaving the injection area for injection. $1 \mathrm{ml}$ of Ethanolamine Oleate was injected into the superior joint space. Then needle withdrawal about $0.5 \mathrm{~cm}$ for injection of $0.5 \mathrm{ml}$ of Ethanolamine Oleate into the peri-capsular tissues (Figure 1).

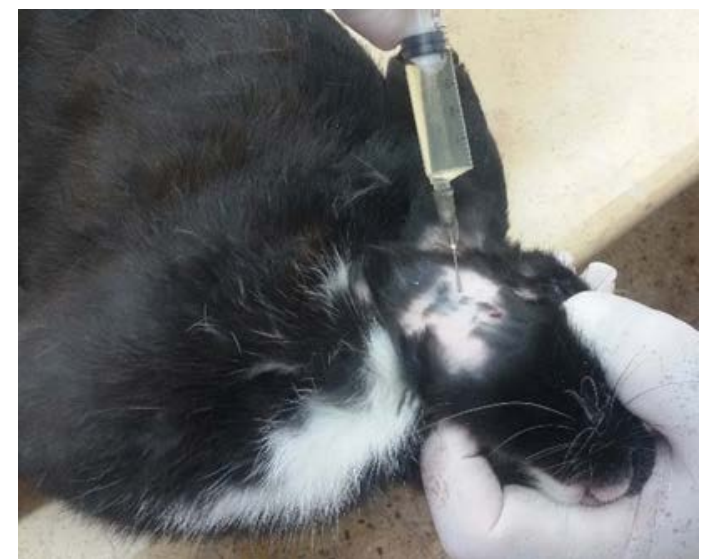

Figure 1: Injection of Ethanolamine Oleate into the joint of TMJ of its capsule.

\section{III- Postoperative phase}

The animals were transferred to a clean cage to be kept under observation to assess the presence or absence of any post-operative complications. Then the animals were maintained on soft diet post-operatively.

\section{IV-Histological evaluation}

A. Tissue of TMJ region (capsular \& pericapsular tissue) had been dissected and immersed immediately in $10 \%$ buffered formalin for 48 hours for fixation.

B. Tissue specimens had been washed under raining tap water for removal of excess formalin. Then the specimens had been dehydrated in ascending grades of alcohol 50\%, $70 \%, 90 \%$ and absolute concentration. Clearance was performed in xylene and finally the specimen had been embedded in blocks paraffin wax to be sectioned in a mesiodistal direction.

C. The paraffin block was serially cut in several sections of $4 \mathrm{~mm}$ thickness and prepared for staining with Hematoxylin and eosin satin (H\&E) for microscopic examination, while other sections were stained with Gomorri stain for the evaluation of fibroblastic activity and collagen formation.

\section{RESULTS}

\section{Histopathologic evaluation}

The pericapsular tissue area surrounding the temporomandibular joint area showed similar histopathologic aspects in all samples. In fact, the pericapsular area showed angiogenesis with presence of fiber, new formed bone and cartilaginous hyaline tissue confirming the diagnosis of fibroblastic activity followed by zone of endochondral ossification and trabecular bone mixed with bone structures.

Two weeks post operatively, the defect areas were filled with an increasingly cellular and granulation tissue with newly formed collagen fibers, they were seen to be distributed haphazardly. This mass of cells appeared to be in continuity with subchondral bone.

\section{Study group}

Histological examination revealed inflammation at the healing area as a reparative nature post operatively.

After one week microscopic features showed fragments of traumatized tissue in the defect area surrounded by blood clot, numerous infiltrating inflammatory cells and profound dilated blood vessels as well as lymphatic spaces.

After 2 weeks, microscopic examination revealed granulation tissue arising from vascular subchondral bone, proliferating fibroblasts and newly formed collagen fibers. 
They were seen to be distributed haphazardly. This cellular proliferating tissue mass appeared to be in continuity with the subchondral bone (Figure 2, 3).

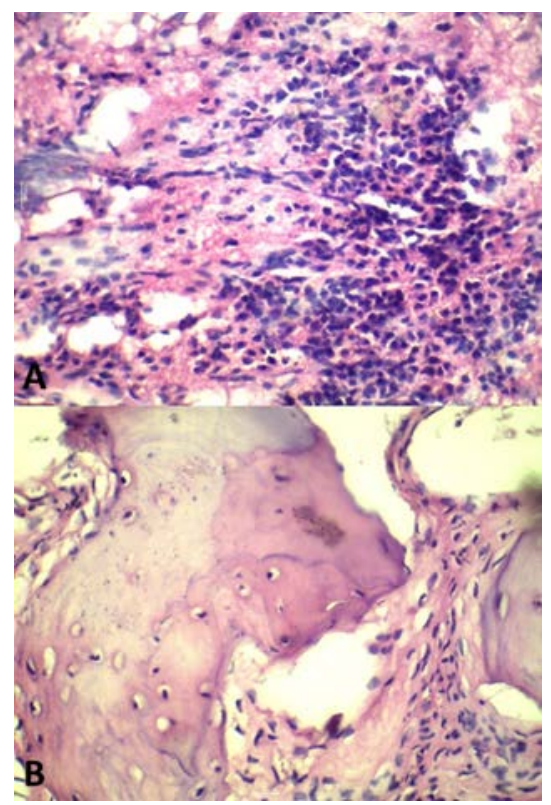

Figure 2: (A) A photomicrograph of a decalcified tissue section (Right side) of a study group 1 week postoperatively showing infiltration of chronic inflammatory cells, blood clot and lymphatic spaces (H\&E*100). (B) A photomicrograph of a study group 2 weeks postoperatively showing vascular spaces and starting proliferating fibroblasts $\left(\mathrm{H} \& \mathrm{E}^{*} 100\right)$.

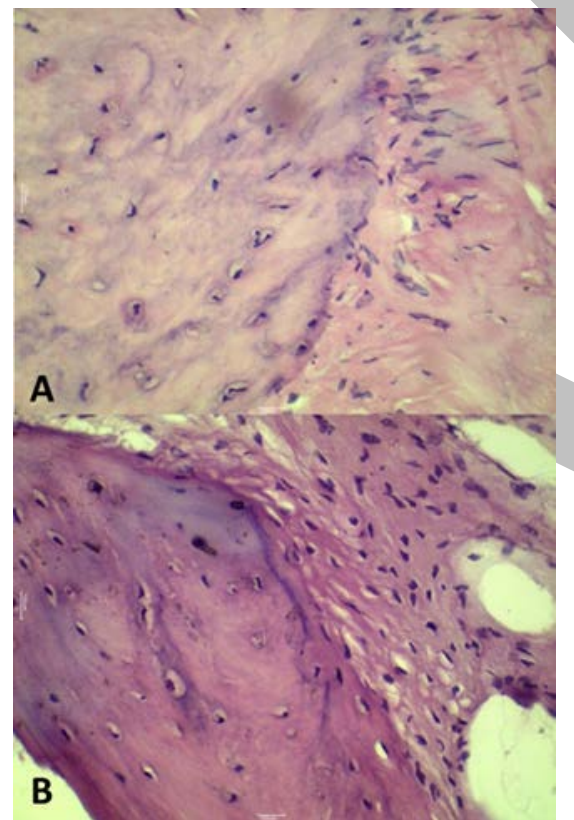

Figure 3: (A) A photomicrograph of a study group 4 weeks postoperatively showing new collagen fibers formation proliferating from defect wound margin revealing the initial signs of organization (H\&E*100). (B) A photomicrograph of another case of a study group postoperatively showing the organized collagen bundles and fibroblastic activity to be in continuity with subcondylar bone (H\&E*100).

The start of reorganization of repairing tissue in the capsular and sub capsular defect area appeared 4 to 6 weeks postoperatively (Figure 4, 5).

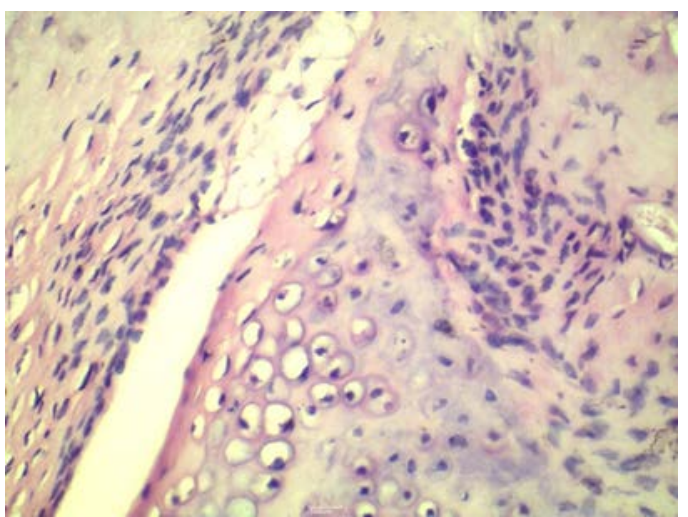

Figure 4: A photomicrograph of a study group 6 weeks postoperatively showing numerous dense fibroblastic activity, hypertrophic chondrocytes and collagen fibers (H\&E*100).

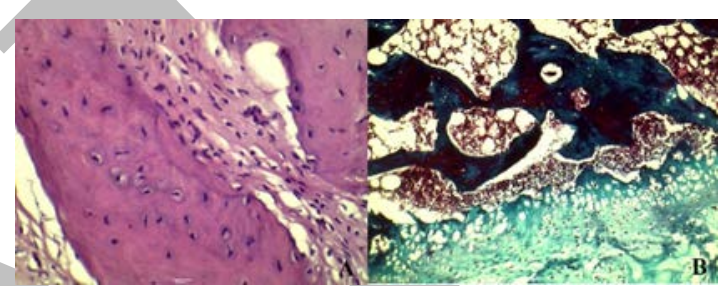

Figure 5: (A) A photomicrograph of another case of a study group 6 weeks postoperatively showing, dense fibroblastic activity and remodeling of both cartilagenous tissue as well as bone activity (H\&E*100). (B) A photomicrograph of a case of a study group 8 weeks postoperatively showing reestablishment of more fibrous tissue hypertrophic chondrocytes and osteoblastic activity within the defect area (Gomorri stain*100).

After 4 to 6 weeks re-establishment of cell layers began and was accompanied by the appearance of labeled cells within cartilage tissue at the margins of the defects area. This cellular activity extended into the zone of repair as a proliferative layer above the fibrous articular zone (Figure 6).

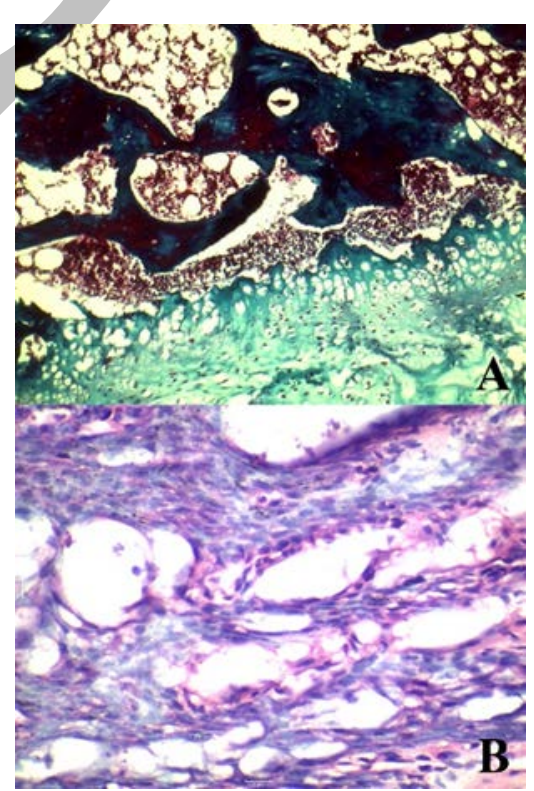

Figure 6: (A) A photomicrograph of a case of a study group 8 weeks postoperatively showing reestablishment of more fibrous tissue hypertrophic chondrocytes and osteoblastic activity within the defect area (Gomorri stain*100). (B) A photomicrograph of a case of control group (left side) showing normal vascular blood vessels and no inflammatory cells (H\&E*100). 
The intercellular matrix was firstly formed of fibrin filling the defect area to be rapidly replaced by collagen fibers which occupied the extent of the defect until separate cell layers were established (collagen fibrillogenesis).

Progressive healing at the articular defect area was formed with hypertrophic chondrocytes and re-established cartilaginous tissue with wounded area.

Some area of neochondrogenesis was seen in specimens between 6-8 weeks. The newly formed cartilage appeared in the subchondral bone around margins of the defect area. Dense collagen fibers were found throughout the articular and proliferative layers as well as in these intercellular areas within the hypertrophic zone in condylar cartilage (Figure 6).

Remodeling of both cartilage and bone at the margins of defect area has been shown to affect the outcome of articular surface repair Osteoblastic activity began to restore the deficiency in subchondral bone making the defect shallower.

Regenerative capacity of condylar cartilage was measured by repairing the standardized full thickness articular defect. Healing wound of the defect area was performed by reconstruction of all tissue elements of condylar cartilage within 6 to 8 weeks of wound defect.

\section{DISCUSSION}

This study was conducted on eight rabbits. Group I (Study Group) underwent injection of $1.5 \mathrm{ml}$ of EO in TMJ capsular and pericapsular tissue in the right side. While group II (control group) included eight rabbits (left side of the same rabbits) was acting as control group. The results of the present study showed a significant fibrosis throughout the follow-up period that expected to limit the jaw movement.

Kato et al (15) presented the management of chronic recurrent TMJ dislocation by autologous blood injection (ABI) in an 84-year-old woman. Three milliliters of autologous blood were injected into the superior joint cavity, and 1 milliliter had been injected into the tissue around the articular capsule of both TMJs. The mandible had been fixed with a bandage, and the use of bandage had been continued for 1 month as a precaution. The result was favorable.

In our experimental study, our explanation for EO is a synthetic mixture of ethanolamine and oleic acid. It acted as a sclerosant when injected intravenously; it acted primarily by irritation of the intimal endothelium of the vein, producing an inflammatory response and fibrosis of the vessel wall. Venous occlusion occurred when EO diffused through the venous wall. The components of EO have different properties. Oleic acid had procoagulant activity. However, EO inhibited the formation of the fibrin clot by chelation of calcium $(16,17)$.

Our results showed that no after-effect complications were reported. However, to date, unfortunately, there had been no experimental analysis on histopathology concerning sclerosing therapies in the literature.

On histological basis, Gulses et al (18) also showed in their study on pigs that $\mathrm{ABI}$ is a safe technique. A total of 16 healthy adult country bred pig where used in their study. Autologous blood was injected into the upper joint space $(4 \mathrm{ml})$ and around the capsule of TMJ unilaterally $(1 \mathrm{ml})$ this procedure was then repeated on the opposite side only by using $5 \mathrm{ml}$ of $0.9 \%$ saline.
While Çandrl et al (19) found that 1 month after the injection the histologic structures were not in a format that satisfied fibrosis and stabilization of the joints. Also, articular cartilage degeneration was not seen in the histologic sections.

Ziegler et al (20) conducted a study on 21 patients with recurrent temporomandibular joint dislocation with results showing, intramuscular injections of botulinum toxin are safe and reliable as a treatment with minimal side effects for recurrent temporomandibular joint dislocations. None of their patients suffered reduced jaw opening, dysphagia, or allergic reaction as results of treatment, and all remained at least dislocation-free. Using Electromyogram, controlled injections of the toxin can be safely placed into the lateral pterygoid muscles. By doing so, the activity of the lateral pterygoid muscle can be reduced and temporarily weakened.

While in our study, only one injection was enough to improve the condition and limit the condylar dislocation which makes the EO more economic and faster.

Platelet rich plasma is also a promising treatment for TMJ dislocation as it is a novel therapeutic agent that has several potential advantages . It accelerates bone and soft tissue healing through delivery of highly concentrated different growth factors and restoring intraarticular hyaluronic acid, increasing glycosaminoglycan chondrocyte synthesis, balances joint angiogenesis and provide scaffold for stem cell migration (21).

As highlighted by Middleton et al (22) Platelet Rich Plasma injections promote optimization of healing environment and facilitate earlier functional rehabilitation of joints.

In a retrospective observational study, Hakala and Ledermann (23) used the technique of dextrose prolotherapy on patients who presented with more than 5 years of unresolved TMJ pain and dysfunction. They demonstrated that prolotherapy was a treatment that can be considered for people with unresolved TMJ pain and dysfunction.

Ongoing discussion about other uses of EA, in the previous literature it was documented as it is a beneficial treatment of pyogenic granuloma and as alternative therapy for vascular lesions in dermatology which is safe and less invasive method with minimal risk. EO also indicated in the treatment of esophageal varices to prevent re-bleeding (13, 24). In human studies it was found that within four days after injection there was neutrophil infiltration of the esophageal wall and hemorrhage within six days. Granulation tissue was first seen at ten days, red thrombi obliterating the varices by twenty days, and sclerosis of the varices by two and a half months. The time course of these findings suggested that sclerosis of esophageal varices was a delay rather than an immediate effect of the drug (25).

\section{CONCLUSION}

Conservative therapy is very important as initial treatment for TMJ dislocation. Ethanolamine Oleate injection is a safe, simple, cost effective treatment of TMJ dislocation with minimal post-operative complication and foreign body reaction.

We encourage injection of Ethanolamine Oleate in the joints for treatment of chronic recurrent TMJ dislocation, as it showed better results. The Ethanolamine Oleate injection to 
the TMJ serves as an alternative \& minimally invasive nonsurgical procedure.

\section{CONFLICT OF INTEREST}

The authors declare that they have no conflicts of interest.

\section{REFERENCES}

1. Miloro M, Ghali GE, Larsen P, Waite P. Peterson's Principles of Oral and Maxillofacial Surgery. 3rd ed. USA: People's Medical Publishing House; 2012.

2. Benoit P. History and physical examination for TMD. Temporomandibular Disorders 2nd ed. New York, NY: Churchill Livingstone; 1994.

3. de Bont LG, Dijkgraaf LC, Stegenga B. Epidemiology and natural progression of articular temporomandibular disorders. Oral Surg Oral Med Oral Pathol Oral Radiol Endod. 1997;83:72-6.

4. Hasson O, Nahlieli O. Autologous blood injection for treatment of recurrent temporomandibular joint dislocation. Oral Surg Oral Med Oral Pathol Oral Radiol Endod. 2001;92:390-3.

5. Fernandez-Sanroman J. Surgical treatment of recurrent mandibular dislocation by augmentation of the articular eminence with cranial bone. J Oral Maxillofac Surg. 1997;55:333-8.

6. Machon V, Abramowicz S, Paska J, Dolwick MF. Autologous blood injection for the treatment of chronic recurrent temporomandibular joint dislocation. J Oral Maxillofac Surg. 2009;67:114-9.

7. Daif ET. Autologous blood injection as a new treatment modality for chronic recurrent temporomandibular joint dislocation. Oral Surg Oral Med Oral Pathol Oral Radiol Endod. 2010;109:31-6.

8. Nitzan DW. Temporomandibular joint “open lock” versus condylar dislocation: signs and symptoms, imaging, treatment, and pathogenesis. J Oral Maxillofac Surg. 2002;60:506-11.

9. Schiffman E, Look J, Hodges J, Swift J, Decker K, Hathaway K, et al. Randomized effectiveness study of four therapeutic strategies for TMJ closed lock. J Dent Res. 2007;86:58-63.

10. Babadag M, Görgün S, Sahin M. Pre-and posttreatment analysis of clinical symptoms of patients with temporomandibular disorders. Quintessence Int. 2004;35:811-4.

11. Aquilina P, Vickers R, McKellar G. Reduction of a chronic bilateral temporomandibular joint dislocation with intermaxillary fixation and botulinum toxin A. Br J Oral Maxillofac Surg. 2004;42:272-3.

12. Matsushita K, Abe T, Fujiwara T. OK-432 (Picibanil) sclerotherapy for recurrent dislocation of the temporomandibular joint in elderly edentulous patients: Case reports. Br J Oral Maxillofac Surg. 2007;45:511-3.

13. Pradhan L, Rahman QB. Effectiveness of Sclerotherapy with Ethanol Amine Oleate in Benign Oral and Perioral Vascular Lesions. BSMMU J. 2011;4:110-5.
14. Kiripolsky MG. Commentary: More on ethanolamine oleate as a vascular sclerosant. Dermatol Surg. 2010;36:1153-4.

15. Kato T, Shimoyama T, Nasu D, Kaneko T, Horie N, Kudo I. Autologous blood injection into the articular cavity for the treatment of recurrent temporomandibular joint dislocation: a case report. J Oral Sci. 2007;49:2379.

16. Da Silva WB, Ribeiro ALR, de Menezes SAF, Pinheiro JdJV, de Melo Alves-Junior S. Oral capillary hemangioma: A clinical protocol of diagnosis and treatment in adults. Oral Maxillofac Surg. 2014;18:4317.

17. Choi YH, Han MH, Kwon OK, Cha SH, Chang KH. Craniofacial cavernous venous malformations: percutaneous sclerotherapy with use of ethanolamine oleate. J Vasc Interv Radiol. 2002;13:475-82.

18. Gulses A, Bayar GR, Aydintug YS, Sencimen M, Erdogan E, Agaoglu R. Histological evaluation of the changes in temporomandibular joint capsule and retrodiscal ligaments following autologous blood injection. J Craniomaxillofac Surg. 2013;41:316-20.

19. Çandrl C, Yüce S, Yldrm S, Sert H. Histopathologic evaluation of autologous blood injection to the temporomandibular joint. J Craniofac Surg. 2011;22:2202-4.

20.Ziegler CM, Haag C, Muhling J. Treatment of recurrent temporomandibular joint dislocation with intramuscular botulinum toxin injection. Clin Oral Investig. 2003;7:52-5.

21. Hanc1 M, Karamese M, Tosun Z, Aktan TM, Duman S, Savaci N. Intra-articular platelet-rich plasma injection for the treatment of temporomandibular disorders and a comparison with arthrocentesis. Jof Craniomaxillofac Surg. 2015;43:162-6.

22. Middleton KK, Barro V, Muller B, Terada S, Fu FH. Evaluation of the effects of platelet-rich plasma (PRP) therapy involved in the healing of sports-related soft tissue injuries. Iowa Orthop J. 2012;32:150.

23. Hakala RV, Ledermann KM. The use of prolotherapy for temporomandibular joint dysfunction. J Prolotherapy. 2010;2:439-46.

24. Hong SK, Lee HJ, Seo JK, Lee D, Hwang SW, Sung HS. Reactive vascular lesions treated using ethanolamine oleate sclerotherapy. Dermatol Surg. 2010;36:1148-52.

25. Alexander MD, McTaggart RA, Choudhri OA, Marcellus ML, Do HM. Percutaneous sclerotherapy with ethanolamine oleate for venous malformations of the head and neck. J Neurointerv Surg. 2014;6:695-8. 